\title{
Dynamic Behavior of Functionally Graded Solid Sphere Subjected To Thermal Load
}

\author{
S.P. Pawar $^{1}$, K.C. Deshmukh ${ }^{2}$, G.D. Kedar ${ }^{2}$ \\ ${ }^{I}$ S. N. Mor and Smt. G. D. Saraf Science College Tumsar Dist-Bhandara (MS), India. \\ ${ }^{2}$ Department of Mathematics, R.T.M. Nagpur University, Nagpur (MS.) India.
}

\begin{abstract}
This article deals with the dynamic transient thermal stresses in a solid sphere of a functionally graded material. The sphere material is considered to be graded along the radial direction, where an exponential varying distribution is assumed. The Poisson's ratio assumed to be constant. The sphere is subjected to a constant temperature at the circular surface of sphere. A numerical finite difference method is used to obtain the time dependent temperature, displacement and thermal stress distribution and results are presented for the FGM sphere consisting $\mathrm{ZrO}_{2}$ and $T_{i}-6 \mathrm{Al}-4 \mathrm{~V}$.
\end{abstract}

Keywords: Transient Thermal stresses, functionally graded material

\section{Introduction}

Functionally graded material is inhomogeneous composites having the properties that vary gradually and continuously within the material with respect to the spatial coordinates. The FGMs concept was first developed by a group of Japanese scientists to meet the need of destructive environment of the thermal shocks and have been widely explored in various engineering applications.FGMs were first used as a shield in industries. New applications have become possible using FGM such as energy conversion, dental and orthopaedic implants, thermogenerators, sensors and joining dissimilar materials (1998).Another important usage of FGM is wear resistant coatings and the covering of mechanical parts such as gears, cams, roller bearings and machine tools (2003).Functionally graded materials are made by combining different material powders by metallurgy methods.

The analytical solution for the stresses on spheres and cylinders made of funcamationally graded material are given by Lutz and Zimmerman $(1996,1999)$. They considered thick sphere and cylinders under radial thermal loads, where radially graded materials with linear composition of constituent materials were considered. Obata and Noda (1994) studied the one dimensional steady thermal stresses in a functionally graded circular hollow cylinder and a hollow sphere using the perturbation method. By introducing the theory of laminated composites, Ootao et al (1995) treated the theoretical analysis of a three dimensional thermal stress problem for a nonhomogeneous hollow circular cylinder due to a moving heat source in the axial direction in a transient state. Tanigawa et al (1999) solved the thermal stresses for a semi infinite body with the assumption that nonhomogeneous material properties are power functions of the thickness direction z. Jabbari et al $(2002,2003)$ derived the analytical solution for one dimensional and two dimensional steady state thermo elastic problem of the functionally graded circular hollow cylinder, where the material properties are expressed as functions of radius. Esalmi et al (2005) obtained an exact solution for the one dimensional steady state thermal and mechanical stresses in a hollow thick sphere of FGM, they assumed the temperature distribution to be a function of radius. Chen and Lin (2008) carried out the elastic analysis for a thick cylinder as well as spherical pressure vessel made of FGM with exponentially varying properties which has significant role in the stress distribution along the radial direction and useful to engineers for design. Shao and Ma (2008) carried out thermo mechanical analysis of FGM hollow cylinder subjected to mechanical loads and linearly increasing boundary temperature .Thermo mechanical properties of functionally graded material are temperature independent and vary continuously in the radial direction of cylinder. Employing Laplace transform techniques and series, solving methods for ordinary differential equations, Tariq Darabseh and Kholoud Bani-Salameh[2010 ] obtained the numerical solution of a FGM cylinder by using implicit finite difference scheme,Nayak and P.Nayak et al(2011) presented an analysis of FGM thick cylinder and spherical vessels with radially varying properties in the form of displacement, strain and stress for thermal mechanical and thermo mechanical loads and validated the method of solution and results by means of reducing it to isotropic and homogeneous material. Recently Farshad Ehsani and Farzad Ehsani (2012) analyzed the one dimensional non steady state temperature distribution in a hollow thick cylinder of FGM with non uniform heat generation by homotopy perturbation method

In this article, the transient thermal stresses in a functionally graded solid sphere subjected to a constant temperature at the surface are obtained by numerical method based on implicit finite difference scheme. The results are illustrated for FGM sphere consists of ceramic $\mathrm{ZrO}_{2}$ and alloy Ti-6A1-4V. In this work an attempt is made to study the behaviour of FGM sphere under thermal load based on uncoupled Thermoelasticity .Under this situation, the thermal and mechanical problems are uncoupled. On determination of the temperature distribution 
inside the sphere from the heat conduction equation, it is regarded as a known function and is introduced in the thermoelastic equations to determine the thermal stresses. The objective of this paper is to provide an effective mathematical model to analyze the transient response for FGM sphere subjected to spherically symmetric thermal load.

\section{Formulation of The Problem}

Consider the FGM solid sphere of radius $a$. The material properties of the sphere are assumed to be function of radius. Assume the material properties of the FGM as a mixture of ceramic and metal vary exponentially in the radial direction as,

$$
k(R)=k_{m} e^{p R}
$$

(1)

$$
\rho(R)=\rho_{m} e^{v R}
$$

(2)

$$
c_{p}(R)=c_{p m} e^{w R}
$$

(3)

In which $p, v$ and $w$ are the dimensionless nonhomogeneity parameters,

$$
\begin{aligned}
& p=\ln \frac{k_{c}}{k_{m}} \\
& v=\ln \frac{\rho_{c}}{\rho_{m}}
\end{aligned}
$$

(4)

$$
w=\ln \frac{c_{p c}}{c_{p m}}
$$

The subscripts $c$ and $m$ means the properties of ceramic and metal respectively. According to (4) the centre of the sphere is metal rich and outer surface is ceramic rich. Following dimensionless parameters are introduced,

$$
\begin{aligned}
& R=\frac{r}{a} \\
& \vartheta=\frac{T-T_{\infty}}{T_{w}-T_{\infty}} \\
& \tau=\frac{t}{t_{0}} \\
& t_{0}=\frac{a^{2} \rho_{m} c_{p m}}{k_{m}}
\end{aligned}
$$

(5)

Where

$a=$ Radius of the sphere

$t_{0}=$ Reference time

$k=$ Thermal conductivity

$T_{w}=$ Surface temperature

$T_{\infty}=$ Ambient temperature

$T=T(r, t)=$ Temperature

$t=$ Time

$\rho=$ Density

$c_{p}=$ Specific heat 


\section{Heat Conduction Problem}

Consider the transient temperature distribution in the FGM sphere. Initially the sphere is assumed to be at uniform temperature and equal to the ambient temperature $T_{\infty}$. The surface of the sphere is maintained at the temperature $T_{w}$. The heat conduction equation without internal heat generation for one dimensional unsteady state distribution of temperature $T(r, t)$ stated in spherical coordinates $(r, \theta, \phi)$ is given as [26],

$$
\frac{\partial^{2} T}{\partial r^{2}}+\frac{\partial T}{\partial r}\left(\frac{2}{r}+\frac{1}{k(r)} \frac{d k}{d r}\right)=\frac{\rho(r) c_{p}(r)}{k(r)} \frac{\partial T}{\partial t}
$$

Subjected to the initial and boundary condition,

$$
T(r, t)=T_{\infty} \quad \text { at } t=0
$$

(7)

$$
T(r, t)=T_{w} \quad \text { at } r=a
$$

(8)

$$
\frac{\partial T}{\partial r}=0 \quad \text { at } \quad r=0
$$

Substituting the material expressions (1-3) and the dimensionless parameters (5), the problem (6-9) is transformed into dimensionless form as,

$$
\frac{\partial^{2} \vartheta}{\partial R^{2}}+\frac{\partial \vartheta}{\partial R}\left(\frac{2}{R}+p\right)=e^{(v+w-p) R} \frac{\partial \vartheta}{\partial \tau}
$$

(10)

The dimensionless initial and boundary conditions are,

$\vartheta(R, \tau)=0 \quad$ at $\tau=0$

(11)

$$
\vartheta(R, \tau)=1 \quad \text { on } \quad R=1
$$

$$
\frac{\partial \vartheta}{\partial R}=0 \quad \text { at } \quad R=0
$$

Setting $p, v$ and $w=0$ the equation reduces to the case of a homogeneous material.

\section{Thermoelasticity problem}

For one dimensional problem in the spherical coordinate system $(r, \theta, \phi)$, which means spherically symmetric problem, the displacement technique is extensively used. The properties in spherical coordinates $\phi$ and $\theta$ direction are identical. The only nonzero components of the displacement vector $u_{r}(r)$ which can be denoted by $u$.The strain displacement relation are as [1],

$$
\begin{gathered}
\varepsilon_{r r}=\frac{\partial u}{\partial r} \\
\varepsilon_{\theta \theta}=\frac{u}{r}
\end{gathered}
$$

(14)

The thermoelastic stress-strain relations are,

$$
\begin{aligned}
& \sigma_{r r}=c_{11} \varepsilon_{r r}+2 c_{12} \varepsilon_{\theta \theta}-\left(c_{11}+2 c_{12}\right) \alpha \Delta T \\
& \sigma_{\theta \theta}=c_{12} \varepsilon_{r r}+\left(c_{11}+c_{12}\right) \varepsilon_{\theta \theta}-\left(c_{11}+2 c_{12}\right) \alpha \Delta T
\end{aligned}
$$

(15)

Where $c_{i j}$ are the elastic constants, $\alpha$, the thermal expansion coefficient , $\Delta T=T-T_{\infty}$

The thermoelastic constants are radially dependent so that $c_{11}=c_{11}(r), c_{12}=c_{12}(r)$ and 
$\alpha=\alpha(r)$.

When the material is isotropic then,

$c_{11}=\frac{E(1-v)}{(1+v)(1-2 v)}=\lambda+2 \mu$

$c_{12}=\frac{E v}{(1+v)(1-2 v)}=\lambda$

$\mu=\frac{E}{2(1+v)}$

(16)

Where $E$ is the Young's modulus and $v$ is Poisson's ratio assumed to be constant.

The stress components in terms of the displacement component $u$, using (14), (15) and (16) may be obtained as [1],

$\sigma_{r r}=\frac{E}{(1+v)(1-2 v)}\left[(1-v) \frac{\partial u}{\partial r}+2 v \frac{u}{r}-(1+v) \alpha \Delta T\right]$

$\sigma_{\theta \theta}=\sigma_{\phi \phi}=\frac{E}{(1+v)(1-2 v)}\left[v \frac{\partial u}{\partial r}+\frac{u}{r}-(1+v) \alpha \Delta T\right]$

(17)

Two of the three equations of stress equilibrium are identically satisfied; the third takes the form as [29, page 277],

$\frac{\partial \sigma_{r r}}{\partial r}+\frac{2}{r}\left(\sigma_{r r}-\sigma_{\theta \theta}\right)=\rho \frac{\partial^{2} u}{\partial t^{2}}$

(18)

By inserting equation (16) in (15) and then in (18) the dynamic equation of motion for displacement $u$ is obtained as,

$\frac{\partial^{2} u}{\partial r^{2}}+\frac{\partial u}{\partial r}\left(\frac{2}{r}+\frac{1}{E} \frac{d E}{d r}\right)+\frac{u}{r}\left(\frac{2 v}{1-v} \frac{1}{E} \frac{d E}{d r}-\frac{2}{r}\right)=\frac{1+v}{1-v}\left[\frac{\partial}{\partial r}(\alpha \Delta T)+\alpha \Delta T \frac{1}{E} \frac{d E}{d r}\right]+\frac{(1+v)(1-2 v)}{(1-v)} \frac{\rho}{E} \frac{\partial^{2} u}{\partial t^{2}}$

The solution of $u$ is known from (19), the stresses can be determined from (17).

It is expected that the stresses at the centre $r=0$ are finite; therefore the displacement $u$ must vanish when $r=0$. The sphere is free of surface tractions. So the initial and boundary conditions becomes,

$u(r, t)=0 \quad t=0$

$\frac{\partial u}{\partial r}=0 \quad t=0$

$u(r, t)=0 \quad r=0$

$\sigma_{r r}(r, t)=0 \quad r=a$

(20)

Introduce the following dimensionless parameters for displacement and stresses as [15],

$U=\frac{u}{a \alpha_{m}\left(T_{w}-T_{\infty}\right)}$

$S_{r}=\frac{\sigma_{r r}}{c_{11 m} \alpha_{m}\left(T_{w}-T_{\infty}\right)}$

$S_{\theta}=S_{\phi}=\frac{\sigma_{\theta \theta}}{c_{11 m} \alpha_{m}\left(T_{w}-T_{\infty}\right)}$ 
The thermoelastic constants $E$ and $\alpha$ are considered as,

$\begin{aligned} E(r) & =E_{m} e^{s R} \\ \alpha(r) & =\alpha_{m} e^{q R}\end{aligned}$

(22)

Where

$s=\ln \frac{E_{c}}{E_{m}}$

$q=\ln \frac{\alpha_{c}}{\alpha_{m}}$

The special case of a homogeneous material is obtained by letting $s, q=0$. The Poisson's ratio $v$ is assumed to be constant. Now by using the parameters in(21), the constants in (22) and the parameters defined in (5),the equation (19) and (17) can be expressed as,

$\frac{d^{2} U}{d R^{2}}+\frac{d U}{d R}\left[\frac{2}{R}+s\right]+\frac{U}{R}\left[\frac{2 v}{1-v} s-\frac{2}{R}\right]=\frac{1+v}{1-v} e^{q R}\left[\frac{d \vartheta}{d R}+\vartheta(q+s)\right]+\frac{(1+v)(1-2 v)}{(1-v)} \frac{\rho_{m} a^{2}}{E_{m} t_{0}{ }^{2}} e^{(v-s) R} \frac{\partial^{2} U}{\partial \tau^{2}}$

$S_{r}=e^{s R}\left[\frac{\partial U}{\partial R}+\frac{2 v}{1-v} \frac{U}{R}\right]-\frac{1+v}{1-v} e^{(s+q) R} \vartheta$

$S_{\theta}=e^{s R}\left[\frac{v}{1-v} \frac{\partial U}{\partial R}+\frac{1}{1-v} \frac{U}{R}\right]-\frac{1+v}{1-v} e^{(s+q) R} \vartheta$

(26)

The dimensionless initial and boundary conditions are,

$U(R, \tau)=0 \quad$ at $\quad \tau=0$
$\frac{\partial U}{\partial \tau}(R, \tau)=0 \quad$ at $\quad \tau=0$
$U(R, \tau)=0 \quad$ at $\quad R=0$
$\frac{\partial U}{\partial R}(1, \tau)+\frac{2 v}{1-v} U(1, \tau)=\frac{1+v}{1-v} e^{q}$

(27)

III. Analytic Solutions

\section{Solution of the Temperature Field}

A fully implicit finite difference scheme is used to evaluate the dimensionless temperature govern by equations (10-13).A backward-difference representation is used for the time derivative and central difference formulas are employed for other derivatives. The implicit schemes are unconditionally stable for any size of time step, but accuracy of the solution is only first-order in time. Hence, small time steps are needed to ensure reasonable accuracy of results.

The differential equation (10) is represented in a finite difference form as,

$$
\frac{\vartheta_{i-1}^{n+1}-2 \vartheta_{i}^{n+1}+\vartheta_{i+1}^{n+1}}{(\Delta R)^{2}}+\frac{\vartheta_{i+1}^{n+1}-\vartheta_{i-1}^{n+1}}{2 \Delta R}\left(\frac{2}{i \Delta R}+p\right)=e^{(v+w-p) i \Delta R} \frac{\vartheta_{i}^{n+1}-\vartheta_{i}^{n}}{\Delta \tau}
$$

Rearranging the terms one gets, 
$\left(\frac{1}{(\Delta R)^{2}}-\frac{1}{(i \Delta R) \Delta R}-\frac{p}{2 \Delta R}\right) \vartheta_{i-1}^{n+1}+\left(-\frac{2}{(\Delta R)^{2}}-\frac{e^{(v+w-p) i \Delta R}}{\Delta \tau}\right) \vartheta_{i}^{n+1}+\left(\frac{1}{(\Delta R)^{2}}+\frac{1}{(i \Delta R) \Delta R}+\frac{p}{2 \Delta R}\right) \vartheta_{i+1}^{n+1}=$

$-\frac{e^{(v+w-p) i \Delta R}}{\Delta \tau} \vartheta_{i}^{n}$

$i=2,3,4,5 \ldots \ldots \ldots . . . N$

(29)

For a solid sphere the equation (10) easily seen singularity at $R=0, R$ and $\frac{\partial \vartheta}{\partial R}$ becomes zero at the node $i=1$

$(R=0)$. Hence we have a $\frac{0}{0}$ ratio at the origin. By application of L'Hospital's rule, it can shown that the ratio has the following determinate form,

$\left(\frac{1}{R} \frac{\partial \vartheta}{\partial R}\right)_{i=1}=\frac{\frac{\partial}{\partial R}\left(\frac{\partial \vartheta}{\partial R}\right)}{\frac{\partial}{\partial R} R}=\left(\frac{\partial^{2} \vartheta}{\partial R^{2}}\right)_{R=0}$

(30)

Thus (10) becomes,

$3 \frac{\partial^{2} \vartheta}{\partial R^{2}}+p \frac{\partial \vartheta}{\partial R}=\frac{\partial \vartheta}{\partial \tau}$

(31)

Additional relationship is obtained by discretizing equation (31) at $R=0$. In order to use a second order accurate central difference formula at $R=0$, a node is needed to the left of the origin $R=0$. This is achieved by considering a fictitious node " 0 " at fictitious temperature $\vartheta_{0}$ located at the distance $\Delta R$ to the left of node $i=1$ on $R$-axis. The resulting finite difference approximation of (31) at $i=1$ becomes,

$3 \frac{\vartheta_{i-1}^{n+1}-2 \vartheta_{i}^{n+1}+\vartheta_{i+1}^{n+1}}{(\Delta R)^{2}}+p \frac{\vartheta_{i+1}^{n+1}-\vartheta_{i-1}^{n+1}}{2 \Delta R}=e^{(v+w-p) i \Delta R} \frac{\vartheta_{i}^{n+1}-\vartheta_{i}^{n}}{\Delta \tau}$

(32)

As the centre of the sphere is metal rich, therefore'

$v=w=p=0$ by $(4)$

Putting $i=1$ in (32) one gets,

$3 \frac{\vartheta_{0}^{n+1}-2 \vartheta_{1}^{n+1}+\vartheta_{2}^{n+1}}{(\Delta R)^{2}}=\frac{\vartheta_{1}^{n+1}-\vartheta_{1}^{n}}{\Delta \tau}$

Where the fictitious temperature $\vartheta_{0}^{n+1}$ is determined by utilizing the symmetry condition at the node $i=1$ as,

$\left(\frac{\partial \vartheta}{\partial R}\right)_{i=1}=\frac{\vartheta_{i+1}^{n+1}-\vartheta_{i-1}^{n+1}}{2 \Delta R}=\frac{\vartheta_{2}^{n+1}-\vartheta_{0}^{n+1}}{2 \Delta R}=0$

(34)

$$
\vartheta_{0}^{n+1}=\vartheta_{2}^{n+1}
$$

Therefore (33) becomes,

$$
3 \frac{\vartheta_{2}^{n+1}-2 \vartheta_{1}^{n+1}+\vartheta_{2}^{n+1}}{(\Delta R)^{2}}=\frac{\vartheta_{1}^{n+1}-\vartheta_{1}^{n}}{\Delta \tau}
$$


$\left(-\frac{6}{(\Delta R)^{2}}-\frac{1}{\Delta \tau}\right) \vartheta_{1}^{n+1}+\frac{6}{(\Delta R)^{2}} \vartheta_{2}^{n+1}=-\frac{1}{\Delta \tau} \vartheta_{1}^{n} \quad$ for $R=0$

Putting $i=N$ in (29) one gets

$\left(\frac{1}{(\Delta R)^{2}}-\frac{1}{(N \Delta R) \Delta R}-\frac{p}{2 \Delta R}\right) \vartheta_{N-1}^{n+1}+\left(-\frac{2}{(\Delta R)^{2}}-\frac{e^{(v+w-p) N \Delta R}}{\Delta \tau}\right) \vartheta_{N}^{n+1}+\left(\frac{1}{(\Delta R)^{2}}+\frac{1}{(N \Delta R) \Delta R}+\frac{p}{2 \Delta R}\right) \vartheta_{N+1}^{n+1}=$ $-\frac{e^{(v+w-p) N \Delta R}}{\Delta \tau} \vartheta_{N}^{n}$

Since $\vartheta_{N+1}^{n+1}=1$ boundary condition

$\left(\frac{1}{(\Delta R)^{2}}-\frac{1}{(N \Delta R) \Delta R}-\frac{p}{2 \Delta R}\right) \vartheta_{N-1}^{n+1}+\left(-\frac{2}{(\Delta R)^{2}}-\frac{e^{(v+w-p) N \Delta R}}{\Delta \tau}\right) \vartheta_{N}^{n+1}=-\frac{e^{(v+w-p) N \Delta R}}{\Delta \tau} \vartheta_{N}^{n}-\left(\frac{1}{(\Delta R)^{2}}+\frac{1}{(N \Delta R) \Delta R}+\frac{p}{2 \Delta R}\right)$

$$
D_{N}=-\frac{e^{(v+w-p) N \Delta R}}{\Delta \tau} \vartheta_{N}^{n}-\left(\frac{1}{(\Delta R)^{2}}+\frac{1}{(N \Delta R) \Delta R}+\frac{p}{2 \Delta R}\right)
$$

Thus the finite difference form of equation (10-13) in matrix form is obtained as,

$$
\left[\begin{array}{ccccccc}
B_{1} & C_{1} & 0 & 0 & 0 & \ldots & 0 \\
A_{2} & B_{2} & C_{2} & 0 & 0 & \ldots & 0 \\
0 & A_{3} & B_{3} & C_{3} & 0 & \ldots & 0 \\
0 & 0 & : & : & : & : & 0 \\
: & : & : & : & : & : & : \\
: & : & : & : & : & : & C_{N-1} \\
0 & 0 & 0 & 0 & 0 & A_{N} & B_{N}
\end{array}\right]\left[\begin{array}{c}
\vartheta_{1}^{n+1} \\
\vartheta_{2}^{n+1} \\
\vartheta_{3}^{n+1} \\
: \\
: \\
\vartheta_{N-1}^{n+1} \\
\vartheta_{N}^{n+1}
\end{array}\right]=\left[\begin{array}{c}
D_{1} \\
D_{2} \\
D_{3} \\
: \\
: \\
D_{N-1} \\
D_{N}
\end{array}\right]
$$

Where,

$$
\begin{aligned}
& B_{1}=-\frac{6}{(\Delta R)^{2}}-\frac{1}{\Delta \tau} \\
& C_{1}=\frac{6}{(\Delta R)^{2}} \\
& D_{1}=-\frac{1}{\Delta \tau} \vartheta_{1}^{n} \\
& A_{i}=\frac{1}{(\Delta R)^{2}}-\frac{1}{(i \Delta R) \Delta R}-\frac{p}{2 \Delta R} \\
& B_{i}=-\frac{2}{(\Delta R)^{2}}-\frac{e^{(v+w-p) i \Delta R}}{\Delta \tau} \\
& C_{i}=\frac{1}{(\Delta R)^{2}}+\frac{1}{(i \Delta R) \Delta R}+\frac{p}{2 \Delta R} \\
& D_{i}=-\frac{e^{(v+w-p) i \Delta R}}{\Delta \tau} \vartheta_{i}^{n} \quad i=2,3,4 \ldots . . N \\
& D_{N}=-\frac{e^{(v+w-p) N \Delta R}}{\Delta \tau} \vartheta_{N}^{n}-\left(\frac{1}{(\Delta R)^{2}}+\frac{1}{(N \Delta R) \Delta R}+\frac{p}{2 \Delta R}\right)
\end{aligned}
$$

The above equations represent a system of $\mathrm{N}+1$ equations with $\mathrm{N}+1$ unknowns for each time step.The resulted matrix is tridigonal. 


\section{Solution of the Thermal Stresses}

A fully implicit scheme is used to evaluate the dimensionless displacement govern by the equations (24) and (27). The backward-difference scheme is used for time derivative, while central difference formulas are employed for other derivatives. The implcit scheme is unconditionally stable for any time step size, but the accuracy of the solution is only first-order in time. Hence, small time steps are needed to ensure reasonable accuracy of results.

The differential equation (24) is represented in a finite difference form as,

$$
\begin{aligned}
& \frac{U_{i-1}^{n+1}-2 U_{i}^{n+1}+U_{i+1}^{n+1}}{(\Delta R)^{2}}+\frac{U_{i+1}^{n+1}-U_{i-1}^{n+1}}{2 \Delta R}\left[\frac{2}{i \Delta R}+s\right]+\frac{U_{i}^{n+1}}{i \Delta R}\left[\frac{2 v}{1-v} s-\frac{2}{i \Delta R}\right] \\
& =\frac{1+v}{1-v} e^{q i \Delta R}\left[\frac{\vartheta_{i+1}^{n+1}-\vartheta_{i-1}^{n+1}}{2 \Delta R}+\vartheta_{i}^{n+1}(q+s)\right]+\frac{(1+v)(1-2 v)}{(1-v)} \frac{\rho_{m} a^{2}}{E_{m} t_{0}^{2}} e^{(v-s) i \Delta R}\left[\frac{U_{i}^{n-1}-2 U_{i}^{n}+U_{i}^{n+1}}{(\Delta \tau)^{2}}\right]
\end{aligned}
$$

Rearranging terms one gets,

$$
\begin{aligned}
& {\left[\frac{1}{(\Delta R)^{2}}-\frac{1}{(i \Delta R) \Delta R}-\frac{s}{2 \Delta R}\right] U_{i-1}^{n+1}-\left[\frac{2}{(\Delta R)^{2}}-\frac{2 v s}{(i \Delta R)(1-v)}+\frac{2}{(i \Delta R)^{2}}+\frac{(1+v)(1-2 v)}{1-v} \frac{\rho_{m} a^{2}}{E_{m} t_{0}^{2}} \frac{e^{(v-s) i \Delta R}}{(\Delta \tau)^{2}}\right] U_{i}^{n+1}} \\
& +\left(\frac{1}{(\Delta R)^{2}}+\frac{1}{(i \Delta R) \Delta R}+\frac{s}{2 \Delta R}\right) U_{i+1}^{n+1}=\frac{1+v}{1-v} e^{q(i \Delta R)}\left[\frac{\vartheta_{i+1}^{n+1}-\vartheta_{i-1}^{n+1}}{2 \Delta R}+(q+s) \vartheta_{i}^{n+1}\right]+\frac{(1+v)(1-2 v)}{(1-v)} \frac{\rho_{m} a^{2}}{E_{m} t_{0}^{2}} \frac{e^{(v-s) i \Delta R}}{(\Delta \tau)^{2}}\left(U_{i}^{n-1}-2 U_{i}^{n}\right)
\end{aligned}
$$

$i=2,3,4, \ldots \ldots . N$

(39)

Putting $i=N+1$ one gets,

$\left[\frac{1}{(\Delta R)^{2}}-\frac{1}{\Delta R}-\frac{s}{2 \Delta R}\right] U_{N}^{n+1}-\left[2+\frac{2}{(\Delta R)^{2}}-\frac{2 v s}{(1-v)}+\frac{(1+v)(1-2 v)}{(1-v)} \frac{\rho_{m} a^{2}}{E_{m} t_{0}^{2}} \frac{e^{(v-s)}}{(\Delta \tau)^{2}}\right] U_{N+1}^{n+1}+$
$\left[\frac{1}{(\Delta R)^{2}}+\frac{1}{\Delta R}+\frac{s}{2 \Delta R}\right] U_{N+2}^{n+1}=\frac{1+v}{1-v} e^{q}\left[\frac{\vartheta_{N+2}^{n+1}-\vartheta_{N}^{n+1}}{2 \Delta R}\right]+\frac{1+v}{1-v}(q+s)+\frac{(1+v)(1-2 v)}{(1-v)} \frac{\rho_{m} a^{2}}{E_{m} t_{o}^{2}} \frac{e^{v-s}}{(\Delta \tau)^{2}}\left(U_{N+1}^{n-1}-U_{N+1}^{n}\right)$

(40)

The value of $U_{N+2}^{n+1}$ and $\vartheta_{N+2}^{n+1}$ can be obtained from (26) and (28), the equation (40) written as,

$$
\begin{aligned}
& \frac{2}{(\Delta R)^{2}} U_{N}^{n+1}-\left[2+\frac{2}{(\Delta R)^{2}}+\frac{4 v}{1-v}\left(1+\frac{1}{\Delta R}\right)+\frac{(1+v)(1-2 v)}{(1-v)} \frac{\rho_{m} a^{2}}{E_{m} t_{o}^{2}} \frac{e^{v-s}}{(\Delta \tau)^{2}}\right] U_{N+1}^{n+1}= \\
& \frac{1+v}{1-v} e^{q}\left[q-2-\frac{2}{\Delta R}\right]+\frac{1+v}{1-v} \frac{2 e^{q}}{\Delta R}\left[\frac{1-\vartheta_{N}^{n+1}}{2+(2+p) \Delta R}\right]+\frac{(1+v)(1-2 v)}{(1-v)} \frac{\rho_{m} a^{2}}{E_{m} t_{0}^{2}} \frac{e^{v-s}}{(\Delta \tau)^{2}}\left[U_{N+1}^{n-1}-2 U_{N+1}^{n}\right]
\end{aligned}
$$

(41)

From this equation one can find the values of $A_{N+1}, B_{N+1}$ and $D_{n+1}$

The finite difference form of equation (24) and (27) in Matrix form is given as,

$$
\left[\begin{array}{ccccccc}
B_{2} & C_{2} & 0 & 0 & 0 & \ldots & 0 \\
A_{3} & B_{3} & C_{3} & 0 & 0 & \ldots & 0 \\
0 & A_{4} & B_{4} & C_{4} & 0 & \ldots & 0 \\
\vdots & \vdots & \ddots & \ddots & \ddots & \ldots & 0 \\
\vdots & \vdots & \vdots & \ddots & \ddots & \ddots & \vdots \\
\vdots & \vdots & \vdots & \vdots & \ddots & \ddots & C_{N} \\
0 & 0 & 0 & 0 & 0 & A_{N+1} & B_{N+1}
\end{array}\right]\left[\begin{array}{c}
U_{2}^{n+1} \\
U_{3}^{n+1} \\
\vdots \\
\vdots \\
\vdots \\
U_{N}^{n+1} \\
U_{N+1}^{n+1}
\end{array}\right]=\left[\begin{array}{c}
D_{2} \\
D_{3} \\
\vdots \\
\vdots \\
\vdots \\
D_{N} \\
D_{N+1}
\end{array}\right]
$$

Where 


$$
\begin{aligned}
& A_{i}=\frac{1}{(\Delta R)^{2}}-\frac{1}{(i \Delta R) \Delta R}-\frac{s}{2 \Delta R} \\
& B_{i}=-\left[\frac{2}{(\Delta R)^{2}}+\frac{2}{(i \Delta R)^{2}}-\frac{2 v s}{(i \Delta R)(1-v)}+\frac{(1+v)(1-2 v)}{(1-v)} \frac{\rho_{m} a^{2}}{E_{m} t_{0}^{2}} \frac{e^{(v-s) i \Delta R}}{(\Delta \tau)^{2}}\right] \\
& C_{i}=\frac{1}{(\Delta R)^{2}}+\frac{1}{(i \Delta R) \Delta R}+\frac{s}{2 \Delta R} \\
& D_{i}=\frac{1+v}{1-v} e^{q(i \Delta R)}\left[\frac{\vartheta_{i+1}^{n+1}-\vartheta_{i-1}^{n+1}}{2 \Delta R}+\vartheta_{i}^{n+1}(q+s)\right]+\frac{(1+v)(1-2 v)}{(1-v)} \frac{\rho_{m} a^{2}}{E_{m} t_{0}^{2}} \frac{e^{(v-s) i \Delta R}}{(\Delta \tau)^{2}}\left[U_{i}^{n-1}-2 U_{i}^{n}\right] \\
& i=2,3,4 \ldots \ldots . . N \\
& A_{N+1}=\frac{2}{(\Delta R)^{2}} \\
& B_{N+1}=-\left[2+\frac{2}{(\Delta R)^{2}}+\frac{4 v}{1-v}\left(1+\frac{1}{\Delta R}\right)+\frac{(1+v)(1-2 v)}{(1-v)} \frac{\rho_{m} a^{2}}{E_{m} t_{0}^{2}} \frac{e^{v-s}}{(\Delta \tau)^{2}}\right] \\
& D_{N+1}=\frac{1+v}{1-v} e^{q}\left(q-2-\frac{2}{\Delta R}\right)+\frac{1+v}{1-v} \frac{2 e^{q}}{\Delta R}\left(\frac{1-\vartheta_{N}^{n+1}}{2+(2+p) \Delta R}\right)+\frac{(1+v)(1-2 v)}{(1-v)} \frac{\rho_{m} a^{2}}{E_{m} t_{0}^{2}} \frac{e^{v-s}}{(\Delta \tau)^{2}}\left(U_{N+1}^{n-1}-U_{N+1}^{n}\right)
\end{aligned}
$$

The components of stress can be calculated from equations (25-26) by using the temperature and displacement histories already obtained. The finite difference form of stress function components at any time for nonzero values of $n$ can be written as,

$$
\begin{aligned}
& S_{r}=e^{s(i \Delta R)}\left[\frac{U_{i+1}^{n}-U_{i-1}^{n}}{2 \Delta R}+\frac{2 v}{1-v} \frac{U_{i}^{n}}{(i \Delta R)}\right]-\frac{1+v}{1-v} e^{(s+q) i \Delta R} \vartheta_{i}^{n} \\
& (43) \\
& S_{\theta}=e^{s(i \Delta R)}\left[\frac{v}{1-v} \frac{U_{i+1}^{n}-U_{i-1}^{n}}{2 \Delta R}+\frac{1}{1-v} \frac{U_{i}^{n}}{(i \Delta R)}\right]-\frac{1+v}{1-v} e^{(s+q) i \Delta R} \vartheta_{i}^{n}
\end{aligned}
$$

$$
i=2,3,4 \ldots . . N
$$

The values of stresses at the centre and on the curve surface of sphere are obtained as follows,

At the centre, $R=0,(i=1)$, by using the L'Hospital's rule the finite difference forms can be obtained as,

$$
S_{r}\left(R=0, \tau_{j}\right)=\left(\frac{1+v}{1-v}\right)\left(\frac{U_{2}^{n}}{\Delta R}-\vartheta_{1}^{n}\right)
$$

$$
S_{\theta}\left(R=0, \tau_{j}\right)=\left(\frac{1+v}{1-v}\right)\left(\frac{U_{2}^{n}}{\Delta R}-\vartheta_{1}^{n}\right)
$$

At the surface, $R=1,(i=N+1)$, the finite difference form of stress components becomes,

$$
S_{r}\left(R=1, \tau_{j}\right)=0 \quad \text { (Traction free surface) }
$$

$$
S_{\theta}\left(R=1, \tau_{j}\right)=e^{s} \frac{\left(1-v-2 v^{2}\right)}{(1-v)^{2}} U_{N+1}^{n}-\frac{(1+v)(1-2 v)}{(1-v)^{2}} e^{s+q}
$$

\section{Results and Discussion}

For the numerical calculations, consider the functionally graded solid sphere has the material properties of ceramic $\mathrm{ZrO}_{2}$ at the heated circular surface $(R=1)$ and alloy

$\mathrm{T}_{\mathrm{i}}-6 \mathrm{~A} 1-4 \mathrm{~V}$.These material composites vary exponentially in the radial direction. The thermo mechanical properties of this material at room temperature are[15], 
Dynamic Behavior Of Functionally Graded Solid Sphere Subjected To Hermal Load

\begin{tabular}{|l|l|l|l|l|l|l|}
\hline Material & $\begin{array}{l}\text { Conductivity } \\
k, W / m K\end{array}$ & $\begin{array}{l}\text { Young's } \\
\text { modulus } \\
E, G P a\end{array}$ & $\begin{array}{l}\text { Coefficient of } \\
\text { thermal expansion } \\
\alpha, 1 / K\end{array}$ & $\begin{array}{l}\text { Poisson's Ratio } \\
v\end{array}$ & $\begin{array}{l}\text { Density } \\
\rho, k g / m^{3}\end{array}$ & $\begin{array}{l}\text { Specific heat } \\
c_{p}, J / k g K\end{array}$ \\
\hline $\begin{array}{l}\text { Metal, } \\
\mathrm{ZrO}_{2}\end{array}$ & 2.09 & 151 & $10 \times 10^{-6}$ & $1 / 3$ & $5.5 \times 10^{3}$ & 418 \\
\hline $\begin{array}{l}\text { Ceramic, } \\
\text { Ti-6A1-4V }\end{array}$ & 7.5 & 116.7 & $9.5 \times 10^{-6}$ & $1 / 3$ & $4.43 \times 10^{3}$ & 560 \\
\hline
\end{tabular}

\section{Set the value of}

$a=1 m, T_{\infty}=0, T_{w}=10 \mathrm{~K}$

The nonhomogeneity parameters for this FGM are

$p=-1.278, v=0.216, w=-0.292, s=0.258, q=0.051$

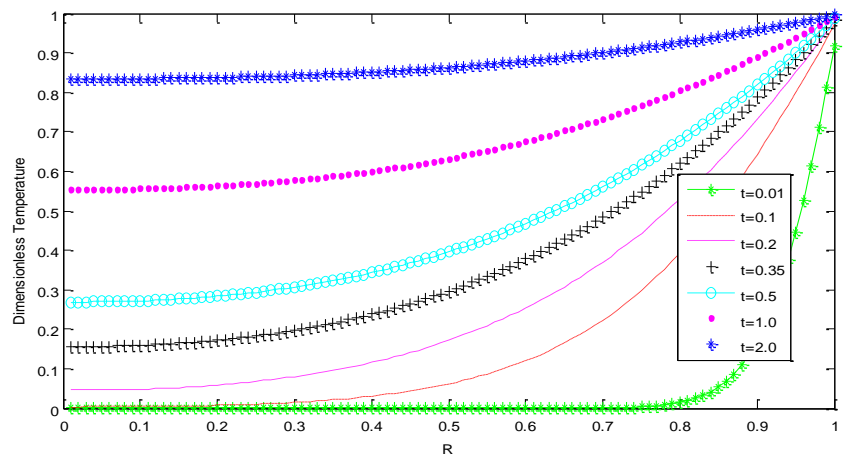

Figure 1: Variation of Dimensionless Temperature along radius

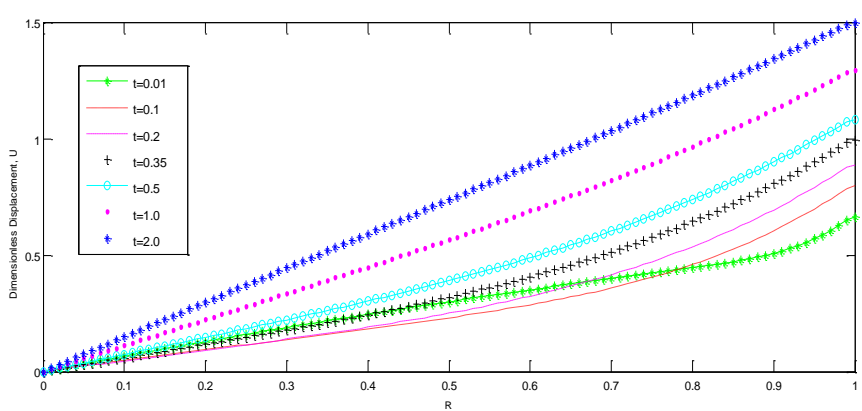

Figure 2: Variation of Dimensionless displacement along radius

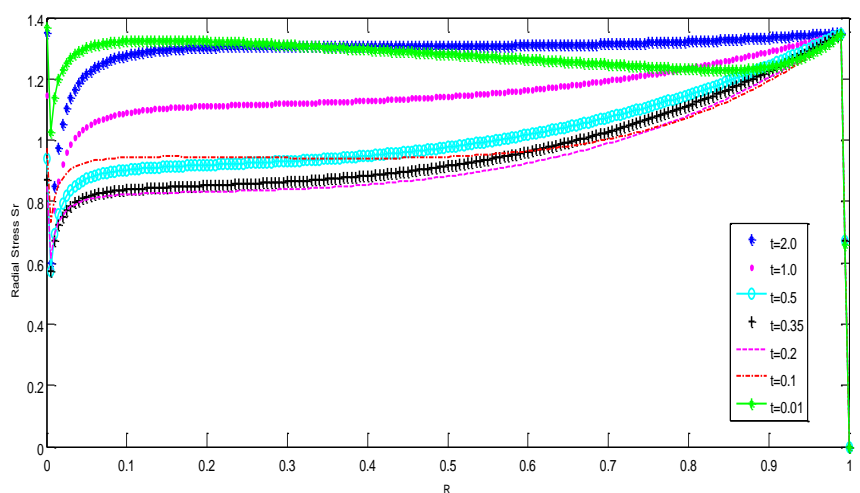

Figure 3: Variation of Dimensionless radial stress along radius 


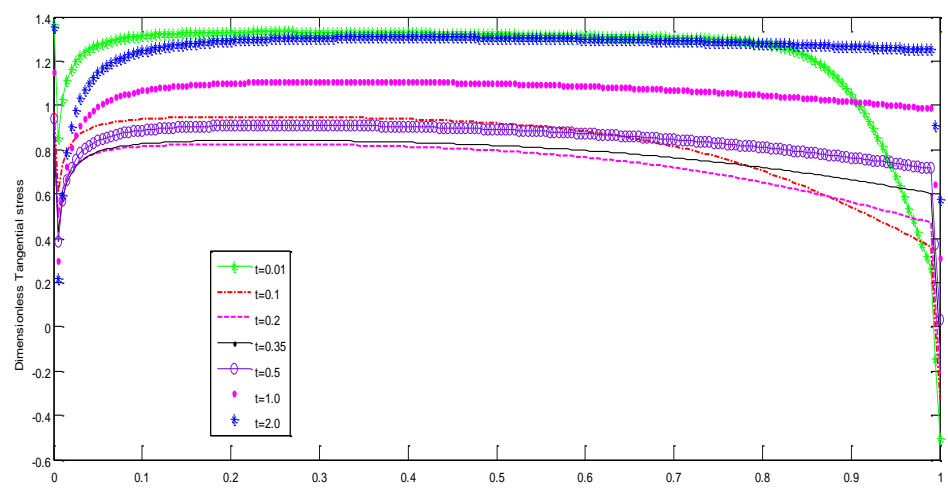

Figure 4: Variation of Dimensionless tangential stress along radius

The ceramic surface of FG sphere is suddenly heated from the initial temperature $T_{\infty}=0$ to $T_{w}=10 \mathrm{~K}$. Fig 1 shows the temperature distribution in the sphere for different values of the dimensionless Fourier time $\tau$ under heating process. For time $\tau=0.01,0.1,0.2,0.35,0.5,1.0,2.0$ the temperature distributions are shown. For lesser values of times the temperatures gradually increase from centre to surface of sphere. As time progresses the temperature value tends towards steady state $\vartheta=1$.For very short time $\tau=0.01$ the variation of temperature is observed near the surface and gradually it shifts towards centre. Fig 2 shows the transient displacement variation along radius of the sphere. In the beginning of heating process the variation in displacement has significant picture and it becomes linear as the time progresses. Fig 3 represents the radial stress variation along radial direction. For time $\tau=0.01$, after sudden change in the surface temperature ,there is a significant variation in radial stress values. Stresses are tensile at the centre and compressive towards surface. As time progresses the stress values distribution approaches to the steady state and extreme stress gradients occur at the heated outer surface. The tangential stress function gives tensile nature throughout in the beginning of heating process. But the nature changes as time progresses. The stresses are tensile at the centre and compressive at the surface. And it approaches to steady state as time progresses further.

\section{Conclusions}

In this work the transient temperature distribution, displacement and thermal stresses are obtained by using fully implicit finite difference scheme. The results are presented for the FGM consists of $\mathrm{ZrO}_{2}$ and $\mathrm{T}_{\mathrm{i}^{-}}$ 6A1-4V.The stresses are computed numerically by using the known temperature obtained independently. The transient results shown are illustrated graphically for $\tau=0.01,0.1,0.2,0.35,0.5,1.0,2.0$.For lesser values of Fourier time $\tau$ the temperature has significant gradient and it reaches to steady state as time progresses further. The displacement has the significant variation for smaller times and it varies linearly as time progresses. Its effect on stresses gives the result which agreements the displacement. For $\tau=0.01$ the radial stress is tensile at the centre and compressive towards the surface, the stresses gives the significant variation for the smaller values of $\tau$..As time progresses the radial stress function change to compression at the surface. And the stresses in region close to the outer surface suddenly drop to zero as per the induced condition on the boundary. The tangential stress function gives tensile nature throughout in the beginning of heating process. . But the nature changes as time progresses. The stresses are tensile at the centre and compressive at the surface. This mathematical model is very useful in engineering field to study the transient thermal stresses in FGM solid spherical tools.

\section{Acknowledgement}

The authors are thankful to University Grants Commission, New Delhi to provide the partial financial assistance under major research project scheme.

\section{References}

[1] Noda N., Hetnarski R.B.and Tanigawa Y., Thermal Stresses, Taylor and Francis, New York, 302, ${ }^{\text {nd }}$ Ed: (2003).

[2] Obata Y. and Noda N., Steady State Thermal stresses in a hollow circular cylinder and a hollow sphere of a functionally graded material, J. Thermal Stresses, 17(3), 471- 487, (1994).

[3] Ootao Y., Akai T. and Tanigawa Y., Three dimensional Transient thermal stresses Analysis of a non-homogeneous hollow circular cylinder due to moving heat source in the axial direction, J. Thermal stresses vol. 18, 497-512,(1995)

[4] Lutz M. P., Zimmerman R. W., Thermal stresses and effective thermal expansion coefficient of A Functionally Graded Sphere, J. Thermal Stresses, 19,39-54, (1996) 
[5] Suresh S. and Mortensen.,Fundamentals of Functionally Graded Materials, Maney, London, U.K., (1998)

[6] Zimmerman R. W. and Lutz M. P., Thermal stresses and effective thermal expansion in a Uniformally heated Functionally Graded Cylinder, J. Thermal Stresses, 22,177- 188, (1999)

[7] Tanigawa Y. Morishita H. and Ogaki S., Derivation of a system of Fundamental Equations for a three dimensional Thermoelastic field with non-homogeneous material properties and its application to a semi-infinite body, J. Thermal stresses, Vol. 22, 689711,(1999).

[8] Jabbari M. Sohrabpour S. and Eslami M. R., Mechanical and thermal stresses in Functionally Graded hollow cylinder due to radially symmetric loads, Int. J. Pressure Vessel Piping, 79, 493-497, (2002).

[9] Jabbari M. Sohrabpour S. and Eslami M. R., General solution for mechanical and thermal Stresses in a functionally graded hollow cylinder due to non axisymmetric steady state Loads, ASME J.appl.Mech., 70,111-118, (2003).

[10] Schulz U., Peters, M. Bach F. W. And Tegeder G., Graded Coatings for Thermal, Wear and Corroosion Barriers Material Science and Engineering A, 362,61-80, (2003).

[11] Eslami M. R. Babaei M. H. and Poultangari R., Thermal and mechanical stresses in a Functionally graded thick sphere , Int. J. Pressure Vessel piping,82,522-527, (2005).

[12] Alavi F. Karimi D. and Bagri .,An investigation on thermo elastic behavior of Functionally graded thick spherical vessels under combined thermal and mechanical Loads, AMME,31(2),(2008)

[13] Chen Y. Z. and Lin X. Y. Elastic analysis for thick cylinders and spherical pressure vessels made of functionally graded materials. Computational Materials Science, 44,581-587,(2008)

[14] Shao Z. S. Ma G. W., Thermo-mechanical stresses in functionally graded circular hollow cylinder with linearly increasing boundary temperature. Composite Structures, 83,259-265, (2008)

[15] Tariq Darabseh and Kholoud Bani-Salameh, Numerical Solution of transient Thermal stresses in a Functionally Graded Engineering Mechanics ,Structures,Engineering Geology,89-96, (2010)

[16] S.Mohammad Reza Khalili,Amir Hossien Mohazzab and Mohsen Jabbari, Anallytical Solution for Two dimensional Magnetothermo-mechanical response in FG Hollow Sphere, Turkish J. Eng. Env.Sci.34,231-252,(2010)

[17] Kulkarni V. S. and Deshmukh K. C., quasi- Static Thermal Stresses in A Thick Circular Plate Due To Axisummetric Heat Supply, Int. J. of Appl. Math and Mech. 5(6):38-50, (2009)

[18] P Nayak. S C Mondal and A Nandi, Stress, Strain and Displacement of a functionally Graded Thick Spherical Vessel, International Journal of Engineering Science and Technology (IJEST), 3,2659-2671, (2011)

[19] Piotr Ostrowski and Bohdan Michalak, Non-stationary heat Transfer in Hollow CylinderWith Functionally Graded material Properties, J. of Theoretical and Applied Mechanics,49, 2,385-397, (2011)

[20] Hasan Çalloğlu, Metin Sayer and Ersin Demir , Stress analysis of functionally graded Discs under mechanical and thermal loads, Indian Journal of Engineering and Materials Sciences, 18,111-118, (2011)

[21] Farshad Ehsani and Farzad Ehsani, Transient heat conduction in Functionally graded thick hollow cylinder under non- uniform heat generation by homotopy perturbation method, J. Basic Appl.Sci. Res., 2 (10) 10676-10685, (2012).

[22] Naotake Noda,Yoshihiro Ootao and Yoshinobu Tanigawa, Transient Thermoelastic Analysis For A Functionally Graded Circular Disk With Piecewise Power Law,J. of Theoritical and Applied mechanics, 50, (3), 831-839, (2012)

[23] Deshmukh K.C, Khandait M.V.and Kulkarni V.S., Thermal deflection due to temperature Distribution in a hollow disk heated by a moving heat source ,Far E J.of Applied Mathematics, 66(1),25-37, (2012)

[24] Kedar G.D. and Deshmukh K.C. ,Determination of Thermal Stresses in A Thin Clamped Hollow Disk under Temperature Field Due to Point heat Source, IOSR Journal of Mathematics (IOSR-JM), 4(6).14-19, (2013)

[25] Ozisik M.N., Boundary value problems of heat conduction, International Textbook Company, Scranton, Pennsylvania, (1968).

[26] Richard B. Hetnarski and M. Reza Eslami, Thermal stresses-Advanced Theory and Applications, Springer, (2009)

[27] Rahimi G. H. and M. Zamini Nezad, Exact solution for thermal stresses in a rotating Thick-walled Cylinder of Functionally Graded Material, Journal of Applied Sciences 8(18), 3276-3272, (2008)

[28] Ozisik M. N., Heat Conduction, Wiley and sons, Inc, $2^{\text {nd }}$ Ed, (1993)

[29] Dhaliwal and Singh, Dynamic Coupled Thermoelasticity,Hindustan Publishing Corp. (India),Delhi-110007,(1980)

[30] Pawar S.P., Deshmukh K. C. and Kedar G. D., Effect of heat generation on quasi-static thermal stresses in a solid sphere.IOSR,Journal of Mathematics,(IOSR-JM),7(3), 63-67,( Aug2013).

[31] Pawar S. P., Deshmukh K. C. and Kedar G. D., Effect of Internal Heat Generation and Temperature Asymmetry in a Hollow Sphere and Its Thermal Stresses. Journal of Thermoelasticity(JOT),1(3), 1-5,(Sept 2013)

\section{Zirconium dioxide-sometimes known as zirconia}

Zirconium dioxide is one of the most studied ceramic materials. Due to its excellent wear resistance, zirconia is used as in thread guide, cams and wire drawing dies. It is also used as a cathode for plasma torches and a nucleating agent for glass ceramics. Zirconia is used as a femoral head component in hip implants. High strength and high toughness allow the hip joint to be made smaller which allows a greater degree of articulation. The ability to be polished to a high surface finish also allows a low friction joint to be manufactured for articulating joints such as the hip.

\section{Titanium Alloy Ti 6Al-4V}

Ti 6Al-4V may be considered in any application where a combination of high strength at low to moderate temperatures, light weight and excellent corrosion resistance are required. Some of the many applications where this alloy has been used include aircraft turbine engine components, aircraft structural components, aerospace fasteners, high-performance automotive parts, marine applications, medical devices, and sports equipment. 